\title{
FUZZY BASED NOVEL CURVATURE FEATURES FOR 3D FINGERPRINT RECOGNITIONS
}

\author{
Gurween Kaur \\ Department of CSE \\ Global Group of Institutes, Amritsar, Punjab, India
}

\begin{abstract}
The essentially the most popular utilized biometrics; fingerprint may be considered for higher than a century. Many effective Fingerprint Recognition techniques usually are proposed while using the rapid progress of fingerprint acquires devices along with the advent associated with countless innovative algorithms. However, they will often be almost based on 2D fingerprint characteristics, even the reality this specific human ring finger is 3D images target. This report has presented an evaluation on different $2 \mathrm{D}$ in addition to $3 \mathrm{D}$ ring finger recognition methods. The review has revealed that the application of 3D fingerprint acceptance is dismissed in most existing investigation. There usually are distortions in addition to deformations released and three dimensional information missing when second fingerprint images are utilized, which are unable to perfectly connect with people's needs in exactness and computational difficulty. The general objective of the dissertation is usually to explore different limitations in existing methods.
\end{abstract}

Keywords- Fingerprint Recognition, Types of fingerprints, Fingerprint Scanner, 3D Fingerprint Image, Curvature Feature Extraction, 3D Reconstruction of Touchless fingerprints.

\section{INTRODUCTION}

Fingerprint Recognition is the process regarding identification while using the thoughts that is created by the little shape formations and actually patterns round the fingertips. No several persons have the exact same agreement regarding shape patterns, and this patterns connected with everyone personal continue being identical throughout lifetime. Fingerprints produce an infallible technique of personal recognition. Different particular features may modify, but fingerprints won't likely. Fingerprints is usually recorded using a typical fingerprint second card or is usually recorded electronically and fed electronically for the FBI created for comparison. By taking a look at fingerprints for the scene of a crime while using the fingerprint report of diagnosed persons, officials can certainly establish utter evidence of the page of personality of a person.
Fingerprint identification, referred to as dactyloscopy or hand print identification, is the method of researching two instances of friction shape skin thoughts (see Minutiae), from human fingers, the side of the hand or even toes, to determine whether these thoughts can came from the exact same personal [9]. The flexibleness of friction shape skin ensures that number two hand or side prints are actually just likewise in every detail; actually two thoughts recorded just after each other from the exact same hand. Fingerprint identification, also known as individualization, requires an expert, or an expert pc system running under limit scoring principles, deciding whether two friction shape thoughts will likely have descends from the exact same hand or palm.

\subsection{Types of Fingerprint}

There are three main types of fingerprints: Obvious prints, latent prints and satisfied prints.

\section{Visible Prints}

Visible Prints are named patent prints and will also be remaining with a couple medium, such as for example body, that shows them to the nude eye. They may be when our body, dirt, printer or even fat about the hand touch a easy area and also leave the friction shape impression which is seen without any development.

\section{Latent Prints}

Latent prints aren't obvious to the naked eye. They're formed from the sweat from sebaceous glands on the body or water, sodium, amino acids and oils found in sweat. The sweat and liquids develop prints should be developed before they is seen or photographed.

\section{Impressed Prints}

Impressed Prints are also referred to as plastic prints and are indentations remaining in soft flexible materials, such as for example clay, feel, color or yet another area which will get the impression. They're apparent and can be looked at or photographed without development. 


\section{International Journal of Engineering Applied Sciences and Technology, 2020 \\ Vol. 5, Issue 6, ISSN No. 2455-2143, Pages 198-204 \\ Published Online October 2020 in IJEAST (http://www.ijeast.com)}

\subsection{Fingerprint Scanner}

The identification or recognition of fingerprints can be extremely beneficial in a variety of situations. To complement fingerprints, a fingerprint scanner could be used. Fingerprint scanners are units that could authenticate personal fingerprints through many different numbers of mathematical operations and alarm designs.

Structure and minutia calculations may be used in a fingerprint scanner. The design algorithm determines the fingerprint's design, which can be certainly one of three: an arc, a trap, or perhaps a whirl. The minutia algorithm evaluates the fingerprint ridges themselves. This examination contains shape endings, or where in fact the ridges conclusion, bifurcation, or wherever ridges separate, and short or dot ridges, which are smaller than the common shape size.

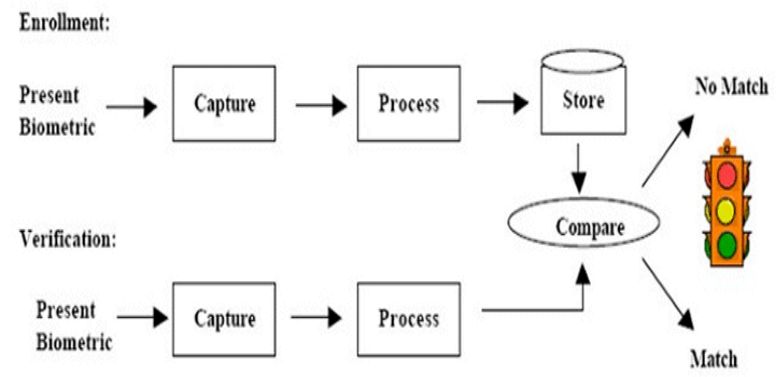

Fig. 1. Fingerprint Scanner

\subsection{D Fingerprint Image}

The fingerprint effect in 3D space, we are able to see which the preceding defined fingerprint functions spread about various machines of level. As an example, core points are situated at the center portion connected with the hand with nearly the most truly effective depth value. Stage two and Degree 3 characteristics which are closely linked to the syndication of ridges really possess more qualities in $3 \mathrm{~d}$ room (e. h. depth value, ridge direction on the depth direction). Hence, in $3 d$ photographs fingerprint picture, characteristics which is often rough than Stage 1 characteristics could possibly be obtained Many of us defined this sort of architectural facts in 3d photographs fingerprint images as Curvature Fingerprint Features with this paper. That curveskeleton function describes this thinned bend of hand shape.

\subsection{Curvature Feature Extraction}

Since our 3D fingerprint picture is reconstructed from multiview fingerprint photographs, there is a one-to-one correspondence between the $3 \mathrm{D}$ points and the $2 \mathrm{D}$ fingerprint picture pixels. Preprocessing such as Location of Curiosity (ROI) removal and pose modification can be done in 2D fingerprint photographs, and executed in 3D situation. Since it's difficult to control the way customers getting their fingers when gathering fingerprint photographs (tilted fingerprint photographs, see Fig. (a)), pose modification is necessary. We achieved it by turning the initial picture the following: i) Check ROI horizontally and find the middle place of every row (green rush point in Fig. (b)); ii) Match such middle points by a point (red strong point in Fig. (b)); iii) Calculate the direction between the fixed point and straight axis $(\theta$ revealed in Fig. (b)); iv) Switch the picture by $\theta$ anticlockwise.

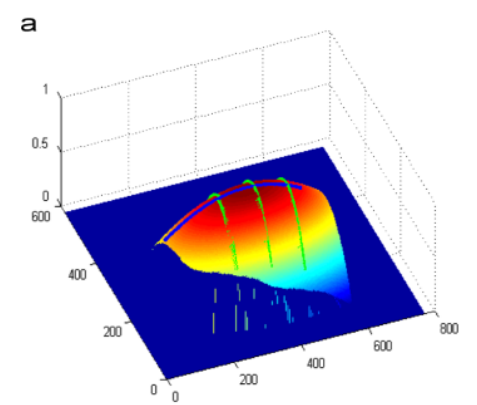

b

Fig. 2. Examples of curve-skeleton for 3D finger. (a) 3D finger shape and (b) extracted curve skeleton. (For interpretation of the references to color in this figure, the reader is referred to the web version of this article.)

\subsection{D Reconstruction of Touchless fingerprints}

Touchless fingerprinting is actually a distant realizing process applied to recapture the ridge-valley pattern. Whilst it is not just a completely new method to obtain fingerprints [2, 3, 4], it did not generate a sufficient interest available in the market, notwithstanding their advantages with respect to the contactbased technology. The key reason is the cost of this technology. In reality, to be able to keep the creation prices of these units minimal, their makers frequently use just one camera. In a Touchless fingerprint picture, the obvious frequency of the ridge-valley design increases from the guts towards the medial side till ridges and valleys become undistinguishable. Thus, devoted methods are essential to correct the ridge- valley design having an upsurge in the entire computational load.

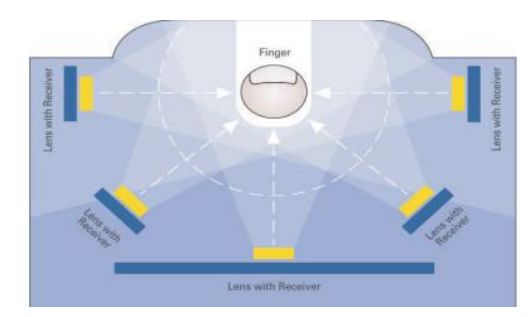

Fig .3 Fingerprint acquisitions using a set of cameras surrounding the finger. 


\section{PROPOSED METHODOLOGY}

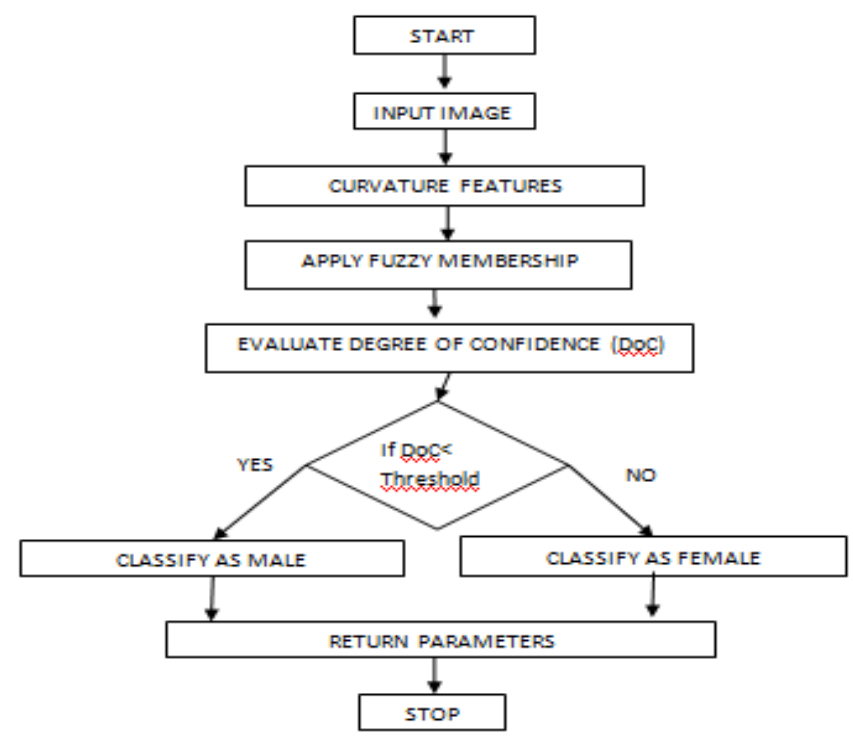

Fig 4. Flow chart of proposed algorithm

The steps of flow chart are discussed below:

Step 1: Start the algorithm.

Step 2: Take any gray scale input image for experimental purposes.

Step 2: Take any gray scale input image for experimental purposes.

Step 3: Apply curvature feature (e.g. curve skeleton and overall maximum curvatures) are used to assist more accurate fingerprint matching or classify human gender after analyzing their characteristics.

Step 4: Then use the Fuzzy logic for improving the accuracy of the novel Curvature Features for 3D fingerprint recognition. Step 5: Then calculate the degree of confidence in order to detect human characteristics. If DOC is less than the threshold value then the human characteristics are male otherwise female.

Step 6: Return parameters are explained.

Step 7: Stop the algorithm.

\section{Proposed Algorithm}

Steps of Proposed Algo:

The detailed algorithm for the proposed approach is given below:

The Fuzzy membership (input_img)

Step 1: Define fuzzy membership requirements

$\mathrm{LSW}=3$;

$\mathrm{PD}=1$;

$\mathrm{FZ}=$ zeros $(\mathrm{LSW} * \mathrm{LSW}, 1)$;

Step 2: Evaluate padding array and size of image input_img $=$

double(padarray(input_img, [padding_value padding_value], 'symm (1)

$[\mathrm{m} \mathrm{n} \sim]=$ size(input_img).

Step 3: Evaluate fuzzy values

for $\mathrm{i}=1$ + padding_value: $1: \mathrm{m}-$

padding_value.

for $\mathrm{j}=1+$ padding_value: $1: \mathrm{n}$ - padding_value

$\mathrm{x}=$ reshape(input_img $(\mathrm{i}-$ padding_value: $\mathrm{i}+$ padding_value, $\mathrm{j}$ - padding_value: $\mathrm{j}+$ padding_value), [],1);.

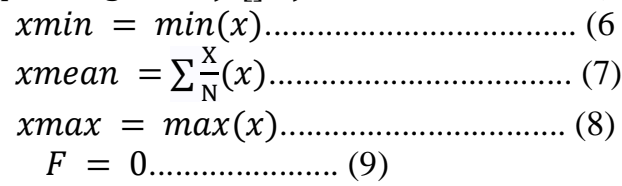

if $(x \operatorname{mean}-x \min ==0) \|(x \max -x \operatorname{mean}==0)$. (10)

$$
\begin{aligned}
& F=1 \\
& \text { Else }
\end{aligned}
$$

$\operatorname{ind} 1=\operatorname{find}((x>=x \min ) \&(x<=x$ mean $)) \ldots .$.

Fuzzy_mem $($ ind 1$)=1-($ xmean $-x($ ind 1$)) /$

$(x m e a n-x \min )$.

ind $2=\operatorname{find}((x>=x$ mean $) \&(x<=x \max )) \ldots . . .(14)$

Fuzzy_mem $($ ind 2$)=1-(x($ ind 2$)-x$ mean $) /(x \max -$ xmean).

End

op_image $(i-$ padding_value,$j-$ padding_value $)=$ $\operatorname{sum}\left(\operatorname{sum}\left(F u z z y \_m e m . * x\right)\right) / \operatorname{sum}\left(\operatorname{sum}\left(F u z z y \_m e m\right)\right) . . . . .$. (16)

End

Step 4: Return membership values as op_image

\section{EXPERIMENT AND RESULT}

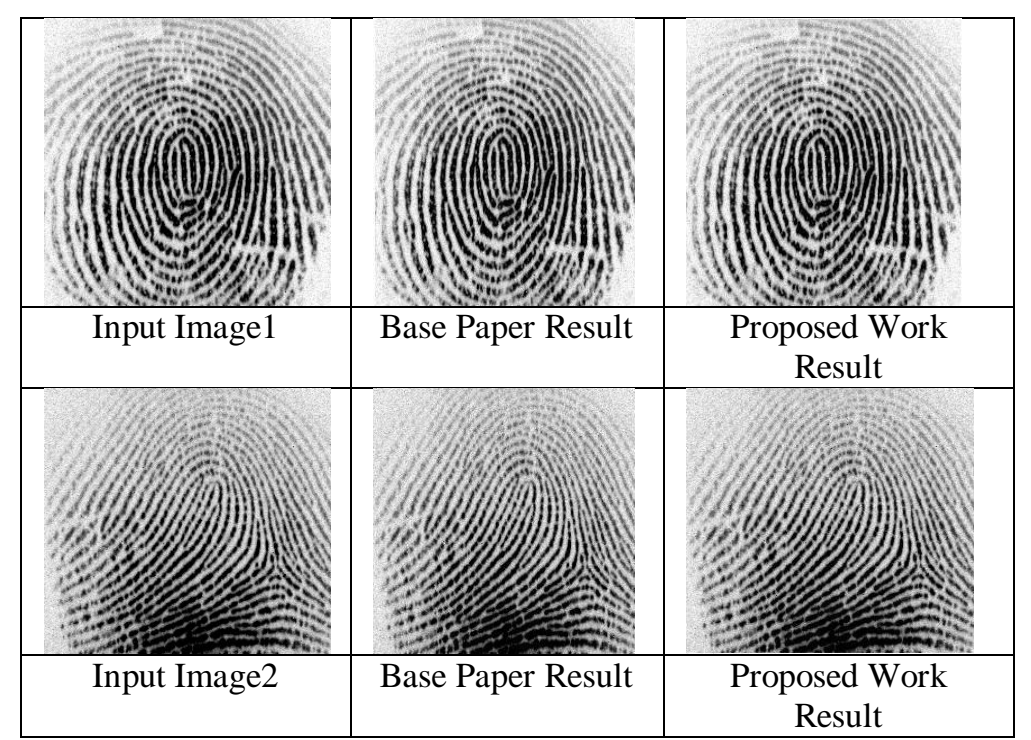




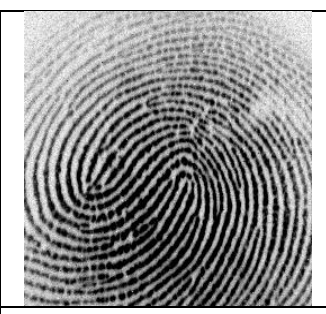

Input Image3

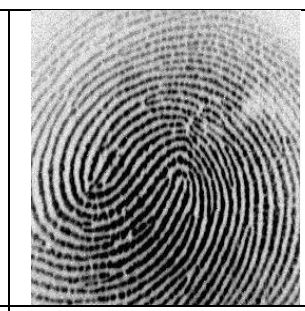

Base Paper Result

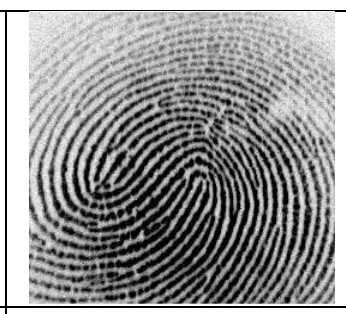

Proposed Work Result

Fig. 5. Experiment Result

Figure 5 Represents the experimental results of different images. This section discusses the various input images. From various input images we find the results of base paper and proposed work. In the base paper we find the results with the help of various curvature features of $3 \mathrm{~d}$ fingerprint recognition but in curvature features results of $3 \mathrm{~d}$ images are almost hide. To overcome this problem we used the fuzzy logic in proposed system. we find the results of proposed system with the help of fuzzy logic for improving the values of all parameters.

\subsection{PERFORMANCE ANALYSIS}

A. F-Measure: F Measure is used for computing the average values of precision and recall. Table 3.1.1 shows the different values of $f$ measure for set of 10 different images. Higher value of $f$ measure is desirable as higher the value of this parameter better will be clustering quality. Table below shows that the proposed technique as higher value than basic algorithm.

Table: 3.1.1 F Measure

\begin{tabular}{|c|c|c|}
\hline S. No. & Existing & Proposed \\
\hline 1 & 88.3763 & 94.9818 \\
\hline 2 & 87.2145 & 94.2873 \\
\hline 3 & 87.7924 & 95.4914 \\
\hline 4 & 92.3185 & 96.8082 \\
\hline 5 & 85.1154 & 97.1796 \\
\hline 6 & 95.8488 & 99.2838 \\
\hline 7 & 95.3250 & 98.2597 \\
\hline 8 & 97.4918 & 99.2609 \\
\hline 9 & 88.9197 & 93.5314 \\
\hline 10 & 95.3736 & 99.6485 \\
\hline
\end{tabular}

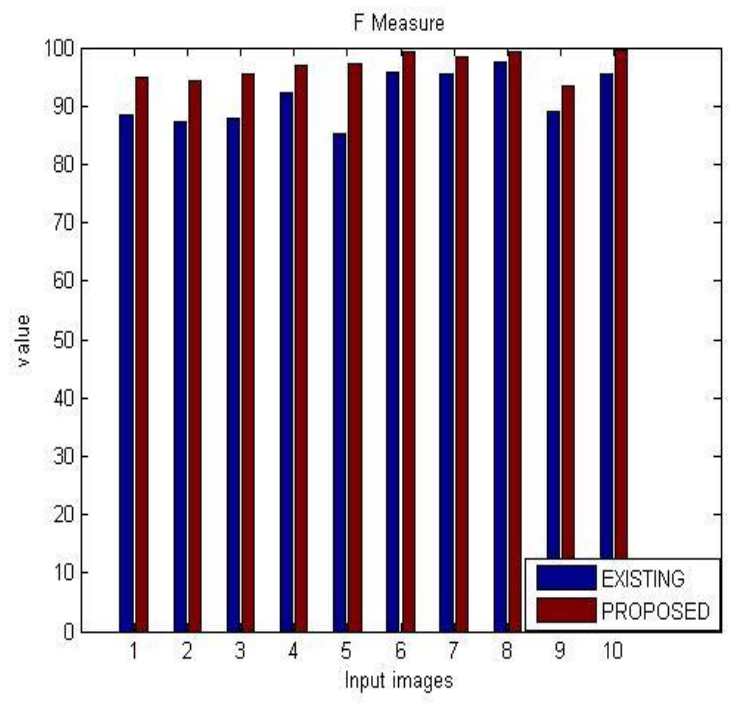

Fig. 6.F measure analysis

Graphical representation of $\mathrm{f}$ measure is shown in figure 6 . From the graph it is clearly illustrated that proposed technique is better than basic lane detection algorithm.

B. Accuracy: Accuracy indicates how properly lane is detected by the algorithm It is also expressed in terms of true positive conditions. Table 3.1.2 shows accuracy values of both the techniques. It shows that accuracy is higher in proposed technique.

Table3.1.2.Accuracy Analysis

\begin{tabular}{|c|c|c|}
\hline S. No. & Existing & Proposed \\
\hline 1 & 0.9253 & 0.9720 \\
\hline 2 & 0.9102 & 0.9657 \\
\hline 3 & 0.8868 & 0.9635 \\
\hline 4 & 0.9331 & 0.9742 \\
\hline 5 & 0.8779 & 0.9800 \\
\hline 6 & 0.9762 & 0.9961 \\
\hline 7 & 0.9626 & 0.9868 \\
\hline 8 & 0.9755 & 0.9955 \\
\hline 9 & 0.9270 & 0.9627 \\
\hline 10 & 0.9880 & 0.9991 \\
\hline
\end{tabular}




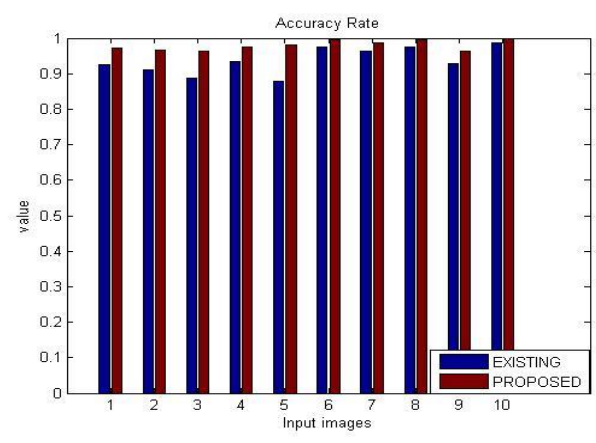

Fig. 7.Accuracy analysis

It is seen from the graph in figure 7 that the proposed technique has higher accuracy than the existing technique. The accuracy of existing technique is shown in blue whereas for proposed red color is used.

C. Bit Error Rate: Bit error rate is defined as number errors that occur in bits divided by the total number of bits transferred in a given period of time. According to need this error rate should decrease and should be minimum. Table 3.1.3 shows the bit error rate measurements of both the techniques.

Table 3.1.3.Bit Error Rate Analysis

\begin{tabular}{|c|c|c|}
\hline S. No. & Existing & Proposed \\
\hline 1 & 10.4133 & 1.7439 \\
\hline 2 & 11.3362 & 1.9793 \\
\hline 3 & 10.8795 & 2.1216 \\
\hline 4 & 7.1336 & 1.8474 \\
\hline 5 & 12.9561 & 2.1638 \\
\hline 6 & 3.9857 & 0.3485 \\
\hline 7 & 4.4662 & 0.7378 \\
\hline 8 & 2.4468 & 0.2565 \\
\hline 9 & 9.9751 & 2.5787 \\
\hline 10 & 4.4218 & 0.1480 \\
\hline
\end{tabular}

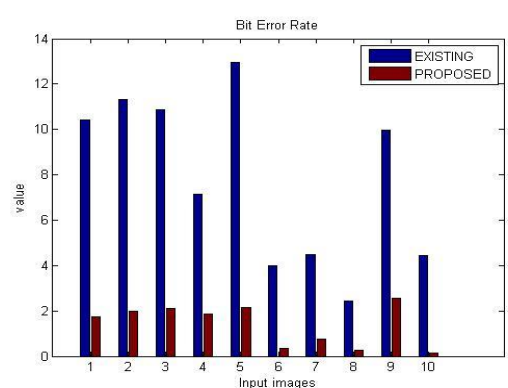

Fig. 8. Bit Error Rate analysis
Figure 8 shows a graph of bit error rate analysis. It is seen that the proposed technique has lower bit error as compared to existing algorithm. Existing values are shown in blue and red represents the values obtained from proposed algorithm. With the decrease in bit error accuracy also increases. This shows that our proposed algorithm is designed well as errors are minimized.

D. Error Rate: Error rate is to consider the errors as the result of adding the data signal to an underlying error signal. The extent of error can then be expressed as the entropy of the error signal, or, in the case of physical signals, as the ratio of the strengths of the two signals - the signal-to-noise ratioexpressed in decibels.

Table 3.1.4: Error Rate

\begin{tabular}{|c|c|c|}
\hline S .No. & Existing & Proposed \\
\hline 1 & 0.0747 & 0.028 \\
\hline 2 & 0.0898 & 0.0343 \\
\hline 3 & 0.1132 & 0.0365 \\
\hline 4 & 0.0669 & 0.0258 \\
\hline 5 & 0.1221 & 0.02 \\
\hline 6 & 0.0238 & 0.0039 \\
\hline 7 & 0.0374 & 0.0132 \\
\hline 8 & 0.0245 & 0.0045 \\
\hline 9 & 0.0730 & 0.0373 \\
\hline 10 & 0.012 & 0.0009 \\
\hline
\end{tabular}

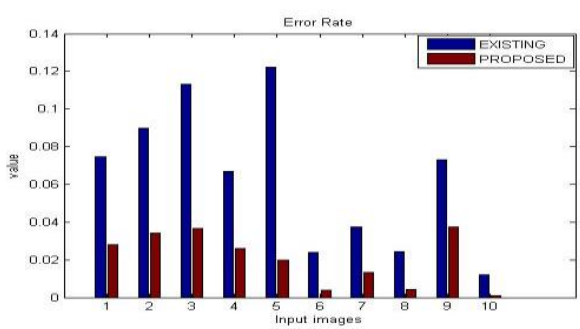

Fig. 9: Error Rate

E. Specificity: Specificity calculates the proportion of negatives that are correctly identified.

Table: 3.1.5 Specificity

\begin{tabular}{|c|c|c|}
\hline S. No. & Existing & Proposed \\
\hline 1 & 0.9963 & 0.9986 \\
\hline 2 & 0.9962 & 0.9985 \\
\hline 3 & 0.9968 & 0.9987 \\
\hline 4 & 0.9975 & 0.9988 \\
\hline
\end{tabular}


International Journal of Engineering Applied Sciences and Technology, 2020

Vol. 5, Issue 6, ISSN No. 2455-2143, Pages 198-204

Published Online October 2020 in IJEAST (http://www.ijeast.com)

\begin{tabular}{|c|c|c|}
\hline 5 & 0.9972 & 0.9990 \\
\hline 6 & 0.9994 & 0.9998 \\
\hline 7 & 0.9973 & 0.9990 \\
\hline 8 & 0.9988 & 0.9996 \\
\hline 9 & 0.9959 & 0.9980 \\
\hline 10 & 0.9984 & 0.9997 \\
\hline
\end{tabular}

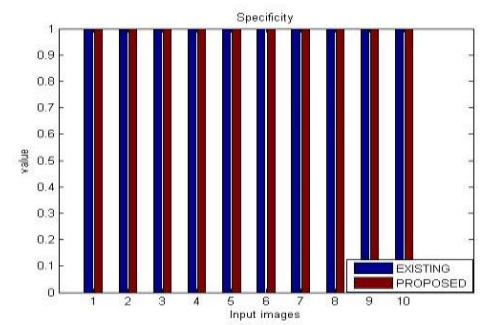

Fig. 10. Specificity

F. Sensitivity: Sensitivity calculates the proportion of positives that are correctly identified.

\section{CONCLUSION}

This dissertation is initialized a review on various $2 \mathrm{D}$ and $3 \mathrm{D}$ fingerprint recognition techniques. In fingerprint recognition you will learn distortions and also deformations introduced and 3 dimensional information misplaced when second fingerprint images are applied, which can't perfectly match with people's specifications in reliability and computational difficulty. The effect of the low intensity of images is also ignored in the majority of existing research. The use of fuzzy logic for better classification of 3D images is also ignored. Fuzzy logic provides two distinctive meanings. From the narrow perception, fuzzy logic is usually a logical method, which is usually an extension linked to multivalued good sense. However, inside a wider perception fuzzy good sense (FL) is nearly synonymous with all the theory linked to fuzzy items, a theory which concerns classes linked to objects along together with unsharp limits where membership can be quite a matter connected to degree. With this perspective, fuzzy good sense in the particular narrow sense is usually a branch linked to FL. Actually inside more filter definition, fuzzy common sense differs both equally within principle and substance received from traditional multivalued reasonable systems. Using this sense, fuzzy logic may be both older and fresh because, although the ultra-modern as well as methodical scientific discipline of fuzzy logic is actually young, the strategy of fuzzy logic is determined by age-old expertise of man reasoning.

Therefore in order to overcome these issues a new fuzzy based novel Curvature Features for 3D fingerprint recognition is proposed in this work. The overall objective of introducing the fuzzy logic is to improve.

\section{REFERENCE}

[1]. Feng Liu, David Zhang and Lenin Shen. (2015). Study on novel Curvature Features for 3D fingerprint recognition, Neurocomputing 168 (pg 599-608).

[2]. Shweta Warade and Rajesh Patil. (May 2015). Touch-less Fingerprint Recognition Using SVM and GMM: A Comparative Study International Journal of Innovative, Research in Computer and Communication Engineering, Vol. 3 , Issue 5 .

[3]. Urvik Patel. (2015). A Study on Fingerprint (biometrics) Recognition, International Journal of Engineering and Sciences, Volume -1 Isuue-2.

[4]. Haryati Jaafar, Salwani Ibrahim, and Dzati Athiar Ramli. (2015). A Robust and Fast Computation Touchless Palm Print Recognition System Using LHEAT and the IFkNCN Classifier, Computational Intelligence and Neuroscience.

[5]. Amrata A. Khindre, V. A. More. (2014). An Approach to Touchless Fingerprint Recognitions using matlab,

Fig. 11 Sensitivity 


\section{International Journal of Engineering Applied Sciences and Technology, 2020 \\ Vol. 5, Issue 6, ISSN No. 2455-2143, Pages 198-204 \\ Published Online October 2020 in IJEAST (http://www.ijeast.com)}

International Journal of Emerging Trends \& Technology in Computer Science.

[6]. Jainam Shah and Ujash Poshiya. (2013). TOUCHLESS FINGERPRINT RECOGNIZATION, Asian Journal of Computer Science and Information Technology 3(5), (pg 73 $-76)$.

[7]. Sangita K Chaudahri. (2012). An algorithm for fingerprint enhancement \& matching, National Conference on Emerging Trends in Engineering \& Technology (VNCET-30).

[8]. Om Preeti Chaurasia. (2012). An Approach to Fingerprint Image Pre-Processing, I.J.Image, Graphics and Signal Processing, 6 (pg 29-35).

[9].Sasan Golabi, Said Saadat, Mohammad Sadegh Helfroush, and Ashkan Tashk. (2012). A Novel Thinning Algorithm with Fingerprint Minutiae Extraction Capability-International Journal of Computer Theory and Engineering, Vol. 4 (4).

[10]. L. Ravi Kumar1, S. Sai Kumar2, J. Rajendra Prasad3, B. V. Subba Rao4, P. Ravi Prakash5.et al. (2012). Fingerprint Minutia Match Using Bifurcation Technique, S Sai Kumar, International Journal of Computer Science \& Communication Networks, Vol 2(4), (pg 478-486).

[11]. Muzhir Shaban Al-Ani et. al. (2012). Face Recognition Approach Based on Wavelet-Curvelet Technique, Signal \& Image Processing: An International Journal (SIPIJ) Vol.3 (2).

[12]. Prabhjot Kaur, Ankit Jain and Sonia Mittal. (2012). Touch-less Fingerprint Analysis - A Review and Comparison, I.J. Intelligent Systems and Applications, 6, (pg 46-52).

[13]. Muzhir Shaban Al-Ani et al. (2011). An Improved Proposed Approach for Hand Written Arabic Signature Recognition Advances in Computer Science and Engineering Volume 7, (1), (Pages 25-35).

[14]. Avinash Pokhriyal et al. (2010) .MERIT: Minutiae Extraction using Rotation Invariant Thinning, International Journal of Engineering Science and Technology Vol. 2(7), (pg 3225-3235).

[15]. ALI H. A. and NE'MA B. M. (2007). Multi-Purpose Code Generation Using Fingerprint Images, 6th WSEAS International Conference on Information Security and Privacy, Tenerife, Spain (pages 14-16). 